\title{
Early strangeness freeze-out from RHIC BES to LHC*
}

\author{
Jia Chen ${ }^{1,2, * *}$, Jian Deng ${ }^{1,2}$, Zebo Tang ${ }^{3}$, Zhangbu $\mathrm{Xu}^{4}$, and $\mathrm{Li} \mathrm{Yi}^{1,2}$ \\ ${ }^{1}$ Institute of Frontier and Interdisciplinary Science, Shandong University, Qingdao, Shandong, 266237, \\ China \\ ${ }^{2}$ Key Laboratory of Particle Physics and Particle Irradiation of Ministry of Education, Shandong Uni- \\ versity, Qingdao, Shandong, 266237, China \\ ${ }^{3}$ State Key Laboratory of Particle Detection and Electronics, University of Science and Technology of \\ China, Hefei, Anhui, 230026, China \\ ${ }^{4}$ Brookhaven National Laboratory, Upton, New York, 11973, USA
}

\begin{abstract}
In this talk, we investigate the collision energy and particle species dependence of kinetic freeze-out properties in relativistic heavy ion collisions from $\sqrt{s_{\mathrm{NN}}}=7.7-200 \mathrm{GeV}$ at Relativistic Heavy Ion Collider (RHIC) and $2.76 \mathrm{TeV}$ at the Large Hadron Collider (LHC) with Tsallis Blast-Wave (TBW) model. Strangeness and non-strange particles show a similar radial flow, while the strange hadrons have higher temperature and smaller non-equilibrium degree. Strangeness approaches equilibrium more quickly than non-strange particles from peripheral to central collisions. The kinetic freeze-out temperature of non-strange particles in central collisions decreases from RHIC to LHC energies, while strangeness does not show this behavior. Finally we discuss the system bulk viscosity dependence on collision energy.
\end{abstract}

\section{Introduction}

The final transverse momentum $\left(p_{T}\right)$ spectra of hadrons carry information about kinetic freeze-out properties of the hot and dense system created in relativistic heavy ion collisions. The study of energy dependence of freeze-out properties is an important tool to explore QCD phase diagram. The RHIC Beam Energy Scan (BES) program and the heavy ion program at the LHC has provided a wide energy coverage for the freeze-out properties study.

Strange hadrons serve as an excellent probe to locate the QCD phase boundary. Specifically strangeness enhancement in heavy ion collisions, compared to elementary $p+p$ collisions, has long been suggested as one of the QGP signatures[1]. Strangeness yields lead to a deeper understanding of strangeness production mechanisms in nuclear collisions[2], and a better constrained extraction of the chemical and kinetic freeze-out parameters. The collision energy dependence of kinetic freeze-out properties for strange hadrons in heavy ion collisions is valuable for exploring QCD phase diagram.

Boltzmann-Gibbs Blast-Wave (BGBW) model [3,4] has been traditionally used to study the produced system at kinetic freeze-out. Tsallis Blast-Wave (TBW) model was later introduced to describe the particle production for an extended $p_{T}$ range in high energy collisions [5-10]. In this proceeding, we will employ blast-wave fits on particle productions from

\footnotetext{
*Presentation based on study in Phys. Rev. C 104, 034901 (2021).

** Speaker. e-mail: jiachen@ rcf.rhic.bnl.gov
} 
RHIC BES to the LHC and focus on the strangeness and non-strangeness kinetic freeze-out comparison.

\section{Analysis method}

In blast-wave model, particle production is assumed to be radiated from local thermal sources. The source is boosted radially with transverse flow profile $\beta$ and is boost-invariant in longitudinal direction. BGBW uses Boltzmann-Gibbs distribution for local thermal equilibrated emission source. The form of particle production in BGBW can be found in [3, 4]. For system deviated from thermal equilibrium, TBW model uses a non-extensive Tsallis statistics for emission source [9-13]. Tsallis statistics introduced a new parameter $q$ for the degree of non-equilibrium, with values larger than 1 for deviation from equilibrium. The produced particle spectrum in TBW is

$$
\left.\frac{d^{2} N}{2 \pi m_{T} d m_{T} d y}\right|_{y=0} \approx \int_{-y_{b}}^{+y_{b}} e^{\sqrt{y_{b}^{2}-y_{s}^{2}}} m_{T} \cosh \left(y_{s}\right) d y_{s} \times \int_{0}^{R} r d r \int_{-\pi}^{\pi}\left[1+\frac{q-1}{T} E_{T}\right]^{-1 /(q-1)} d \phi,
$$

where $E_{T}=m_{T} \cosh \left(y_{s}\right) \cosh (\rho)-p_{T} \sinh (\rho) \cos (\phi)$, with $y_{s}$ and $y_{b}$ for source and beam rapidity.

\section{Results and discussions}
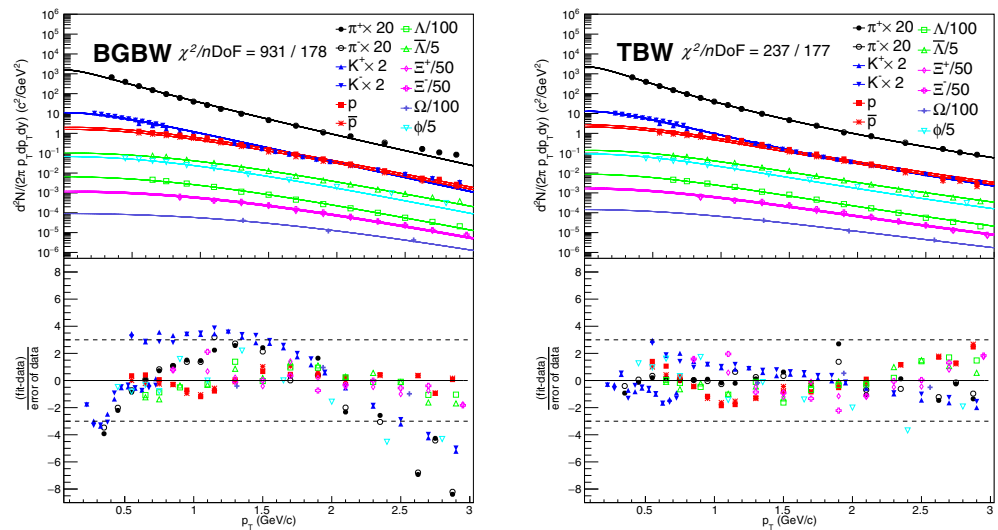

Figure 1. BGBW (left column) and TBW (right column) fits to hadron spectra (top panels) and model deviations from data divided by data uncertainties (bottom panels) in $40 \%-60 \% \mathrm{Au}+\mathrm{Au}$ collisions at $\sqrt{s_{\mathrm{NN}}}=200 \mathrm{GeV}$. The solid curves represent fit functions.

The charged hadron spectra for RHIC $\mathrm{Au}+\mathrm{Au}$ collision data of 7.7-200 GeV and the $\mathrm{LHC} \mathrm{Pb}+\mathrm{Pb}$ collision data of $2.76 \mathrm{TeV}$ are used in this study. Spectra fit range is $p_{T} \leqslant$ $3 \mathrm{GeV} / c$ for a comparable $p_{T}$ range across all energies while focusing on the bulk properties. Figure 1 shows blast-wave fits to identified particle transverse momentum spectra in $40 \%$ $60 \% \mathrm{Au}+\mathrm{Au}$ collisions at $\sqrt{s_{\mathrm{NN}}}=200 \mathrm{GeV}$ in top panels, with corresponding deviations of those fits to experimental data divided by data uncertainties shown in the bottom panels. BGBW and TBW models are compared in the left and right column of Fig.1. TBW agrees with most data points within $3 \sigma$. With smaller $\chi^{2} / n$ DoF, TBW gives a better description for 


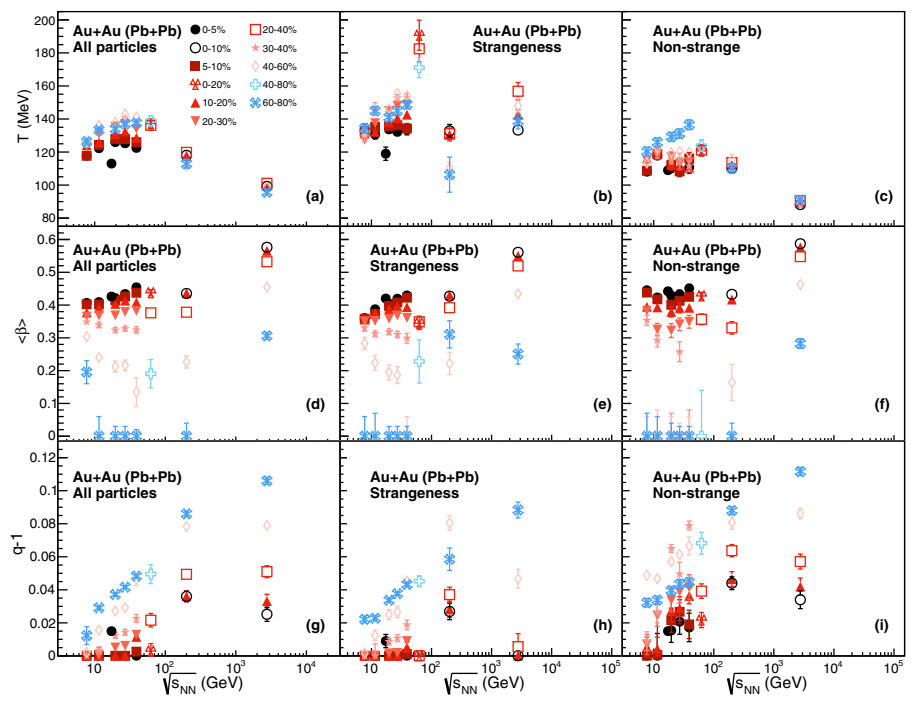

Figure 2. Collision energy dependence of the TBW extracted kinetic freeze-out parameters in different centralities for all particles (left column), strangeness (middle column), and non-strange particles (right column).

data than BGBW fit. Figure 2 shows the energy and centrality dependence of kinetic freezeout parameters temperature $T$, radial flow velocity $\langle\beta\rangle$ and the degree of non-equilibrium $(q-$ 1 ) of the TBW model. Temperature $T$ shows an increasing trend with beam energy at RHIC BES energy, then decreases toward LHC energy. In central collisions, radial flow velocity $\langle\beta\rangle$ increases as collision energy increases. At any given collision energy, $\langle\beta\rangle$ decreases from central to peripheral collisions. The degree of non-equilibrium $(q-1)$ increases from central to peripheral and with increasing collision energy. In central collisions at RHIC BES energy, $(q-1)$ is close to 0 . Smaller $\langle\beta\rangle$ with smaller $(q-1)$ at lower collision energy suggests that a weaker expansion with smaller deviation from thermal equilibrium, or energy density fluctuations at initial state either decrease or are more washed out at later stage at those lower collision energies. A higher $T$ and smaller $\langle\beta\rangle$ for RHIC than LHC suggests the system at RHIC reaches freeze-out earlier with a lower radial flow than at LHC.

The comparison of strangeness and non-strange in the middle and right columns of Fig.2 shows that the strange hadrons have smaller non-equilibrium degree $(q-1)$ but higher temperature $T$, with similar radial flow $\langle\beta\rangle$ than their non-strange partners. It suggests that strangeness freeze-out earlier and approach equilibrium quickly from peripheral to central collisions than light hadrons. Non-strange hadrons exhibit a higher kinetic freeze-out temperature at RHIC than LHC. However, strange hadrons consistently show similar freeze-out temperatures for RHIC and LHC. This difference indicates that the system at RHIC spends shorter time for non-strange particles to reach kinetic freeze-out than at LHC.

Bulk viscosity measures damping for volumetric expansion in fluid. It has been suggested that the bulk viscosity increases dramatically toward the phase transition [14]. In non-equilibrium statistics framework, the dependence of temperature on the non-equilibrium degree is associated with the bulk viscosity $\xi$ in linear or quadratic proportion [5]. We extract information about $\xi$ from the quadratic fits of $T=T_{0}+b(q-1)-d \xi(q-1)^{2}$ for each centrality 


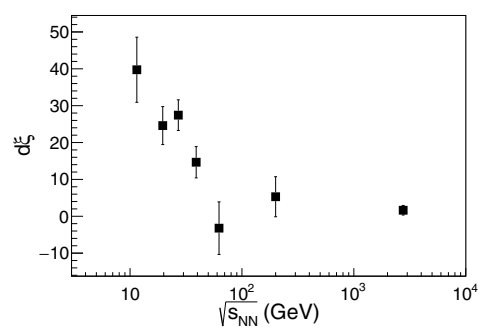

Figure 3. Collision energy dependence of bulk viscosity related parameters $d \xi$.

class at each collision energy, where $d \xi, T_{0}$ and $b$ are fitting paramaters. We observed that $d \xi$, the term associated with bulk viscosity, indeed increases toward lower collision energies.

\section{Conclusion}

We present a study of BGBW and TBW fits on spectra of $\mathrm{Au}+\mathrm{Au}$ (RHIC) collisions at $\sqrt{s_{\mathrm{NN}}}=7.7-200 \mathrm{GeV}$, and $\mathrm{Pb}+\mathrm{Pb}(\mathrm{LHC})$ collisions of $\sqrt{s_{\mathrm{NN}}}=2.76 \mathrm{TeV}$. We find $\mathrm{TBW}$ describes experimental measurements better. With TBW, $T$ shows an increasing trend with beam energy at RHIC BES, then decreases toward LHC. $\langle\beta\rangle$ and $q-1$ increase as collision energy increases. Strange particles freeze out earlier than light hadrons and approach equilibrium more quickly from peripheral to central collisions. The system at RHIC spends shorter time for non-strange particles to reach kinetic freeze-out than that at LHC. Bulk viscosity related parameter increases quickly toward lower collision energies.

This work was partly supported by the National Natural Science Foundation of China under Grants No. 11890710, No.11890713 and No.11720101001.

\section{References}

[1] J. Rafelski and B. Muller, Phys. Rev. Lett. 48 (1982), 1066 [erratum: Phys. Rev. Lett. 56 (1986), 2334]

[2] J. Adam et al. [STAR Collaboration], Phys. Rev. C 102, 034909 (2020)

[3] E. Schnedermann, J. Sollfrank and U. W. Heinz, Phys. Rev. C 48, 2462 (1993)

[4] E. Schnedermann and U. W. Heinz, Phys. Rev. C 50, 1675 (1994)

[5] G. Wilk and Z. Wlodarczyk, Eur. Phys. J. A 40, 299 (2009)

[6] W. M. Alberico, A. Lavagno and P. Quarati, Eur. Phys. J. C 12, 499 (2000)

[7] T. Osada and G. Wilk, Phys. Rev. C 77, 044903 (2008) Erratum: [Phys. Rev. C 78, 069903 (2008)]

[8] T. S. Biro and B. Muller, Phys. Lett. B 578, 78 (2004)

[9] K. Jiang et al., Phys. Rev. C 91, 024910 (2015)

[10] Z. Tang, Y. Xu, L. Ruan, G. van Buren, F. Wang and Z. Xu, Phys. Rev. C 79, 051901 (2009)

[11] K. Urmossy, G. G. Barnaföldi, S. Harangozó, T. S. Biró and Z. Xu, J. Phys. Conf. Ser. 805, no.1, 012010 (2017)

[12] M. Shao, L. Yi, Z. Tang, H. Chen, C. Li and Z. Xu, J. Phys. G 37, 085104 (2010)

[13] Z. Tang et al., Chin. Phys. Lett. 30, 031201 (2013)

[14] F. Karsch, D. Kharzeev and K. Tuchin, Phys. Lett. B 663, 217-221 (2008) 